\title{
Light-induced melatonin suppression: age-related reduction in response to short wavelength light
}

\author{
Mirela Herljevic, Benita Middleton, Kavita Thapan, Debra J. Skene* \\ Centre for Chronobiology, School of Biomedical and Molecular Sciences, University of Surrey, Guildford, Surrey GU2 7XH, UK
}

Received 30 July 2004; received in revised form 15 December 2004; accepted 17 December 2004

Available online 5 January 2005

\begin{abstract}
One of the possible causes of disturbed circadian rhythms and sleep in the elderly may be impaired photic input to the circadian clock. Age-related changes in lens density are known to reduce the transmission of short wavelength light, which has been shown to be most effective in suppressing nocturnal melatonin. The aim of the study therefore was to investigate age-related changes in melatonin suppression in response to short and medium wavelength light.

Young premenopausal $(n=13)$ and postmenopausal $(n=21)$ women were exposed to 30 min of monochromatic light at two different wavelengths and irradiances $\left(\lambda_{\max } 456 \mathrm{~nm}: 3.8\right.$ and $9.8 \mu \mathrm{W} / \mathrm{cm}^{2} ; \lambda_{\max } 548 \mathrm{~nm}$ : 28 and $\left.62 \mu \mathrm{W} / \mathrm{cm}^{2}\right)$. Melatonin suppression was compared across light treatments and between age groups.

Significantly reduced melatonin suppression was noted in the elderly subjects following exposure to short wavelength (456 $\mathrm{nm})$ light compared to the young subjects. These results are likely to reflect age-related changes in lens density.

(C) 2004 Elsevier Inc. All rights reserved.
\end{abstract}

\section{Introduction}

The spectral sensitivity of the ability of light to suppress nocturnal melatonin in humans has been investigated and two action spectra constructed (Brainard et al., 2001; Thapan et al., 2001). These action spectra show distinct short wavelength sensitivity, identifying $457-464 \mathrm{~nm}$ as the most potent wavelength in suppressing nocturnal melatonin synthesis. Recent research also demonstrates that the ability of light to phase advance (Warman et al., 2003) and phase delay (Lockley et al., 2003) circadian rhythms shows a similar short wavelength sensitivity.

Many studies have reported age-related alterations in different components of the circadian system (reviewed by Van Someren, 2000; Skene and Swaab, 2003). Of particular relevance to age-related changes in photic entrainment are changes in the ageing eye. There are a number of age-related changes in the eye that may contribute to reduced levels of

\footnotetext{
* Corresponding author. Tel.: +44 1483 689706; fax: +44 1483300374 .

E-mail address: d.skene@surrey.ac.uk (D.J. Skene).
}

light reaching the retina, such as the reduction in pupil size (Verriest, 1971) and changes within the crystalline lens (Weale, 1985). These changes would reduce the amount of light input to the circadian clock located in the suprachiasmatic nuclei $(\mathrm{SCN})$ of the hypothalamus. Within the crystalline lens accumulation of high molecular weight crystallin aggregates (Jedziniak et al., 1973; Yu et al., 1985) and yellow chromophores (Dillon and Atherton, 1990) cause an increase in light scattering and light absorption, respectively. As a result the density of the lens increases with ageing (Weale, 1954; Coren and Girgus, 1972; Xu et al., 1997) causing an alteration in the spectral absorption. The greatest increase in absorption occurs at the short wavelength end of the spectrum (around 400-470 nm) (Said and Weale, 1959; Norren and Vos, 1974; Smith and Pokorny, 1975). Furthermore with ageing, the lens darkens and develops a yellow pigmentation that further reduces light transmission to the retina (Lerman et al., 1976; Garner et al., 1999). These age-related changes in the eye may affect photic entrainment of the circadian clock and may in part explain some of the changes in circadian 
rhythmicity and in the sleep/wake cycle observed in the elderly (Bliwise, 1993; Duffy et al., 1998; Dijk and Duffy, 1999; Ancoli-Israel, 2000).

As short wavelength light has been found to be the most effective in suppressing nocturnal plasma melatonin (Brainard et al., 2001; Thapan et al., 2001) and as transmission of this short wavelength light is reduced with ageing, it might be expected that the melatonin response to short wavelength light is reduced in older people. This hypothesis, however, has not yet been tested. A short report (Nathan et al., 1999) showed that suppression of melatonin by exposure to 200 lux white light was not affected by age. In this study, no significant difference was noted in melatonin suppression between three age groups, the oldest age group being $41.7 \pm 2$ years (mean \pm SEM). To date, however, the effects of monochromatic short wavelength light on melatonin suppression have not been studied in the elderly.

The aim of the present study was to investigate the effect of age on the ability of short $(456 \mathrm{~nm})$ and medium $(548 \mathrm{~nm})$ wavelength light to suppress nocturnal melatonin.

If the melatonin response is dependent on the age-related change in the spectral density of the lens, then it would be expected that there would be a significantly reduced response to the short wavelength light in the older group, whereas the response to the medium wavelength light would not differ significantly between the two age groups. Light intensities were chosen to give submaximal suppression of nocturnal plasma melatonin, according to our earlier data (Thapan et al., 2001).

\section{Methods}

\subsection{Subjects}

In total, 41 subjects were recruited onto the study but four subjects withdrew during the study and three subjects had to be excluded from the data analysis due to low, undetectable plasma melatonin levels. Two groups of subjects, young ( $n=13$, aged $24 \pm 3$ years, mean \pm SD) and postmenopausal ( $n=21$, aged $57 \pm 5$ years) women were included in the analysis. All subjects gave informed consent and the University of Surrey Ethics Committee approved the study.

The menopausal status of the postmenopausal women was defined by a minimum of 12 months without menstruation and was confirmed by blood hormone analysis (follicle stimulating hormone 22-153 IU/L, lutenising hormone $11-40 \mathrm{IU} / \mathrm{L}$, oestradiol $<200 \mathrm{pmol} / \mathrm{L}$ ). All subjects were free of any medication known to affect the melatonin rhythm ( $\beta$-blockers, calcium channel blockers, $\alpha$-blockers, non steroidal anti-inflammatory drugs, benzodiazepines, antidepressants, hypnotic drugs, antipsychotics, barbiturates and antiepileptic drugs). All young subjects were taking oral contraceptives. None of the postmenopausal women were taking hormone replacement therapy
(HRT). Subjects underwent an extensive ophthalmological examination and were free of ocular disorders (glaucoma, cataracts, colour defects).

\subsection{Study protocol}

Preceding the clinical laboratory study, the subjects' endogenous melatonin rhythm was assessed by measuring urinary 6-sulphatoxymelatonin (aMT6s). The time of light administration was standardised so that each individual received light on the rising phase of their endogenous melatonin rhythm. This was estimated from an individual's aMT6s peak time, light being given $2.5-4.5 \mathrm{~h}$ before the aMT6s peak. Three days before commencement of the clinical study subjects were asked to keep a regular sleep-wake schedule (23:00-07:00 h). Daily sleep diaries and monitoring of the subjects' activity and light exposure by Actiwatch recordings (AWL, Cambridge Neurotechnology Ltd, UK) confirmed compliance. The subjects were instructed to avoid alcohol, caffeine, excessive exercise and bright light $24 \mathrm{~h}$ before the clinical study to minimise factors known to affect circadian phase.

The study consisted of two 3-night sessions separated by at least 5 days. Night 1 (N1) of each session was a baseline night (18:00-07:00 h) with no light exposure. Blood samples for dim light melatonin onset (DLMO) were collected every $30 \mathrm{~min}$ on N1 (20:00-23:00 h). The baseline night (N1) was followed by two light exposure nights (N2 and N3) with each subject being exposed to $30 \mathrm{~min}$ of monochromatic light at two different wavelengths and irradiances $\left(\lambda_{\max } 456 \mathrm{~nm}: 3.8\right.$ and $9.8 \mu \mathrm{W} / \mathrm{cm}^{2} ; \lambda_{\max }$ $548 \mathrm{~nm}: 28$ and $62 \mu \mathrm{W} / \mathrm{cm}^{2}$ ). On N2 subjects were exposed to the lower irradiance of the selected light wavelength followed by the higher irradiance on N3. The same 3-night protocol was repeated in a second session with the other light wavelength. The order of light wavelength exposure $\left(\lambda_{\max } 456\right.$ or $\left.548 \mathrm{~nm}\right)$ was randomised between subjects.

Posture and environmental light were controlled throughout the study (N1-19:00-23:00 h; N2 and N3-21:00-23:00 h: dim light $<5$ lux: 2 lux in the angle of gaze and 4-5 lux when looking directly at the overhead white lights; 23:00-07:00 h: 0 lux, complete darkness with subjects wearing eye masks). Pupil size was controlled with one drop of a short acting pupil dilator, Tropicamide $0.5 \%$ (Chauvin Pharmaceuticals, Romford, UK) administered to each eye $90 \mathrm{~min}$ before the light exposure. On each night of the study session (N1-N3) blood samples were taken via an indwelling cannula at $-90,-15,0,15,30,45,60,75,90$ and $120 \mathrm{~min}$ around the light exposure ( 0 min was onset of light exposure).

\subsection{Sample analysis}

Plasma melatonin was assessed by radioimmunoassay (Fraser et al., 1983) with reagents supplied by Stockgrand Ltd (University of Surrey, Guildford, UK). 
The limit of detection for the plasma melatonin assay was $4.6 \pm 0.2 \mathrm{pg} / \mathrm{ml}$ (mean \pm SEM). The interassay coefficients of variation $(\mathrm{CV})$ were $15 \%$ at $26 \mathrm{pg} / \mathrm{ml}(n=46), 18 \%$ at $54 \mathrm{pg} / \mathrm{ml}(n=38), 14 \%$ at $139 \mathrm{pg} / \mathrm{ml}(n=27)$ and $9 \%$ at $235 \mathrm{pg} / \mathrm{ml}(n=12)$.

\subsection{Light exposure}

The light pulse was produced by a metal halide (a mixture of metal halide with high doses of InI and T1I) arc lamp housed in a fan cooling box (Enlightened Technology Associates Inc., Fairfax, USA). This produced a beam of white light that was delivered to a sphere $(45 \mathrm{~cm}$ diameter) via a fibre optic cable. The white light was filtered to a specific wavelength using monochromatic filters. The visible bandpass monochromatic filters (Coherent Ealing, Watford, UK) were constructed from multi-cavity dielectric on borosilicate glass. The spectral quality of the monochromatic filters was altered slightly from the manufacturer's specifications due to the design of the sphere. Spectroradiometer (Spectrascan 650, Photoresearch, Chatsworth, CA, USA) measurements confirmed the $\lambda_{\max }$ wavelengths at eye level to be 456 and $548 \mathrm{~nm}$ instead of 460 and $560 \mathrm{~nm}$, respectively. Subjects were exposed to the monochromatic light (full width at half maximum $(\mathrm{FWHM}) \leq 13 \mathrm{~nm}$ ) by placing their heads into the sphere. The sphere was coated with white reflectance paint (Kodak, Hemel Hempstead, UK) providing constant uniform illumination of the entire retina. An adjustable chin rest and head band ensured a fixed eye level with the gaze directed to a dot centred at the back of the sphere. Light irradiance $\left(\mu \mathrm{W} / \mathrm{cm}^{2}\right)$ was measured at the subject's eye level (optical powermeter, Macam Photometrics, Livingstone, UK). Each irradiance measured was corrected for the specific light wavelength using calibrated correction factors $(456 \mathrm{~nm}$ : 0.836 and $548 \mathrm{~nm}$ : 0.982). Subjects were exposed to two different wavelengths and irradiances of light $\left(\lambda_{\max } 456 \mathrm{~nm}\right.$ : 3.8 and $9.8 \mu \mathrm{W} / \mathrm{cm}^{2} ; \lambda_{\max }$ $548 \mathrm{~nm}$ : 28 and $62 \mu \mathrm{W} / \mathrm{cm}^{2}$ ) of different photon densities $\left(\lambda_{\max } 456 \mathrm{~nm}: 8.7 \times 10^{12}\right.$ and $2.2 \times 10^{13}$ photons $/ \mathrm{cm}^{2} / \mathrm{s} ; \lambda_{\max }$ $548 \mathrm{~nm}: 7.7 \times 10^{13}$ and $1.7 \times 10^{14}$ photons $/ \mathrm{cm}^{2} / \mathrm{s}$ ).

\subsection{Data analysis}

Suppression of plasma melatonin (\%) was calculated by comparing the mean melatonin concentrations at 30 and 45 min after commencement of light administration (points of maximum melatonin suppression) with the melatonin concentration before light administration $(0 \mathrm{~min})$. The area under the curve (AUC) of the percentage melatonin suppression (0-45 min) was also calculated. Two-way repeated measures analysis of variance (ANOVA) was used to determine the effect of light treatment and age on melatonin suppression (Statistica, Statsoft Ltd, Bedford, UK). In view of our hypothesis (age-related changes in lens density would reduce the response to $456 \mathrm{~nm}$ light but not to $548 \mathrm{~nm}$ light) the two wavelengths of light were analysed separately. Significant results were further analysed post hoc using Tukey Honestly Significant Difference (HSD) test. Two postmenopausal subjects had to be excluded from the analysis due to missing blood samples for one of the light conditions. Due to poor repeatability of the melatonin profile between the baseline night and light treatment nights (15/32 subjects had baseline values differing by more than $20 \%$ in at least one light condition), baseline N1 values were not included in the calculation of melatonin suppression as this would produce an inaccurate estimation of melatonin suppression.

Average spectral lens density calculations for each individual were done in accordance with the Pokorny et al. (1987) two-factor non-linear lens density spectrum model. In this model, the optical density of the total lens transmission $\left(T_{\mathrm{L}}\right)$ is separated into two components which are light-wavelength dependent, (i) portion of the lens affected by ageing after the age of $20\left(T_{\mathrm{L} 1}\right)$ and (ii) stable portion after the age of $20\left(T_{\mathrm{L} 2}\right)$. Using this model average spectral lens density was estimated for adults aged 20-80 years for completely open $(>7 \mathrm{~mm})$ pupils.

\section{Results}

Preceding the clinical study, analysis of urinary aMT6s showed that the young women had significantly higher $24 \mathrm{~h}$ aMT6s production $(22.6 \pm 2.6 \mu \mathrm{g} / 24 \mathrm{~h}$, mean \pm SEM) compared to the postmenopausal women $(12.4 \pm 1.5 \mu \mathrm{g} / 24 \mathrm{~h}$ $p<0.05$, unpaired Student's $t$-test). Furthermore, the postmenopausal women had significantly earlier aMT6s peak times $(4.0 \pm 0.3 \mathrm{~h})$ than the young women $(5.0 \pm 0.2 \mathrm{~h}$, $p<0.05)$.

Fig. 1 shows the time course of melatonin suppression in the young and postmenopausal women following the four different light treatments. Maximal suppression of melatonin occurred 30 and $45 \mathrm{~min}$ after the start of light exposure. Melatonin levels returned to baseline values 30-60 min after lights off depending on the intensity of the light.

Melatonin suppression (mean \pm SEM) for each light treatment is plotted in Fig. 2. There was a significant effect of age and light condition on melatonin suppression. Twoway repeated measures ANOVA revealed a significant effect of both age $\left(F_{(1,30)}=5.80, p=0.02\right)$ and $456 \mathrm{~nm}$ light $\left(F_{(1,30)}=11.83, p=0.002\right)$ on melatonin suppression. Tukey HSD post hoc test revealed a significant reduction in melatonin suppression in the postmenopausal subjects following both $3.8 \mu \mathrm{W} / \mathrm{cm}^{2}(-1.4 \pm 5 \%)$ and $9.8 \mu \mathrm{W} / \mathrm{cm}^{2}$ (12.5 $\pm 4 \%) 456 \mathrm{~nm}$ light ( $p<0.001$ and 0.05 , respectively) compared to melatonin suppression following the same light irradiances (18.4 \pm 4.8 and $25.5 \pm 6.4 \%$, respectively) in young subjects (Fig. 2). No interaction between age and light irradiance with the $456 \mathrm{~nm}$ light was observed $\left(F_{(1,30)}=1.25\right.$, $p=0.27)$. In contrast there was no significant effect of age $\left(F_{(1,30)}=2.17, p=0.15\right)$, light irradiance $\left(F_{(1,30)}=2.89\right.$, $p=1)$ or interaction $\left(F_{(1,30)}=2.90, p=1\right)$ with the $548 \mathrm{~nm}$ 


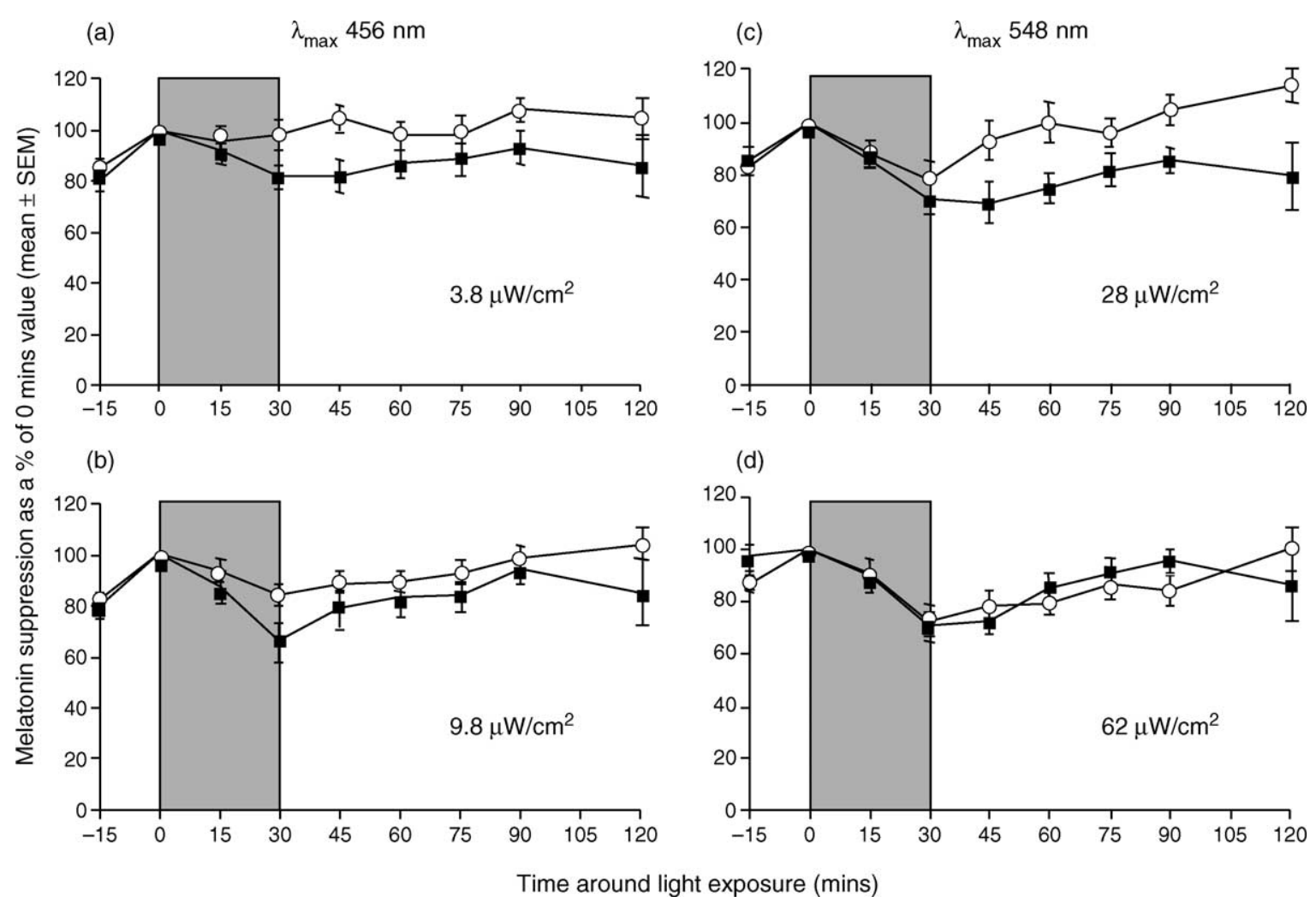

Fig. 1. Melatonin suppression expressed as a percentage of the 0-point value (time of lights on) for young $(\boldsymbol{\square})$ and postmenopausal $(\bigcirc)$ subjects across the four different light treatments: (a) $3.8 \mu \mathrm{W} / \mathrm{cm}^{2} \lambda_{\max } 456 \mathrm{~nm}$, (b) $9.8 \mu \mathrm{W} / \mathrm{cm}^{2} \lambda_{\max } 456 \mathrm{~nm}$, (c) $28 \mu \mathrm{W} / \mathrm{cm}^{2} \lambda_{\max } 548 \mathrm{~nm}$ and (d) $62 \mu \mathrm{W} / \mathrm{cm}^{2} \lambda_{\max }$ $548 \mathrm{~nm}$. $\square$ Light exposure.

light. Similar results were obtained from the AUC 0-45 min analysis. Two-way repeated measures ANOVA of AUC $0-45$ min showed an effect of both age $\left(F_{(1,30)}=4.43, p=\right.$ $0.04)$ and $456 \mathrm{~nm}$ light treatment $\left(F_{(1,30)}=9.82, p=0.004\right)$ on melatonin suppression without interaction $\left(F_{(1,30)}=0.55\right.$, $p=0.46)$. Tukey HSD post hoc test revealed a significant reduction in melatonin suppression (AUC $0-45 \mathrm{~min}$ ) in the postmenopausal subjects following $3.8 \mu \mathrm{W} / \mathrm{cm}^{2} 456 \mathrm{~nm}$ light $(p<0.01)$ and a non significant trend following $9.8 \mu \mathrm{W} / \mathrm{cm}^{2} 456 \mathrm{~nm}$ light $(p=0.07)$ compared to the young subjects. Furthermore, no effect of age $\left(F_{(1,30)}=0.50, p=\right.$ $0.48)$, light irradiance $\left(F_{(1,30)}=0.29, p=0.59\right)$ or interaction $\left(F_{(1,30)}=0.35, p=0.55\right)$ following exposure to $548 \mathrm{~nm}$ light was noted.

A significant negative correlation was found between an individual's spectral lens density and melatonin suppression following exposure to $456 \mathrm{~nm}$ light of 3.8 and $9.8 \mu \mathrm{W} / \mathrm{cm}^{2}$ $\left(F_{(2,29)}=3.87\right.$, multiple $\left.r=-0.45 ; p<0.05\right)$ using multiple regression analysis. No significant correlation was noted between an individual's spectral lens density and melatonin suppression following exposure to $548 \mathrm{~nm}$ light.

\section{Discussion}

This is the first demonstration that the ability of short wavelength light to suppress melatonin is reduced with age.
Although age-related changes in lens density with consequent reduction in the transmission of short wavelength light has been well characterised (Said and Weale, 1959; Norren and Vos, 1974; Smith and Pokorny, 1975), this is the first time that this phenomenon has been linked to a physiological non-image forming light response i.e. melatonin suppression. Melatonin suppression was only significantly different between the two age groups following exposure to short wavelength light. Both decreased pupil diameter (Verriest, 1971) and opacification of the ageing lens (Weale, 1954; Coren and Girgus, 1972; Xu et al., 1997) may contribute to the reduced amount of short wavelength light reaching the retina. Ideally, knowledge of the lens density of each study subject would be useful so that the data could be individually corrected. Using the two-factor non-linear lens density spectrum formula (Pokorny et al., 1987) the average lens density was calculated for each subject. Lens density was negatively correlated with melatonin suppression following short wavelength treatments. There was no correlation between lens density and melatonin suppression following medium wavelength light exposure.

Poor repeatability of the melatonin rhythm between the study nights was observed in 15 of the 32 subjects. The reason for this is not clear. There was no consistent pattern to the change in melatonin concentrations between the study nights. Previous studies using the current protocol (Thapan et al., 2001) did not observe this variability in the young, 
(a)

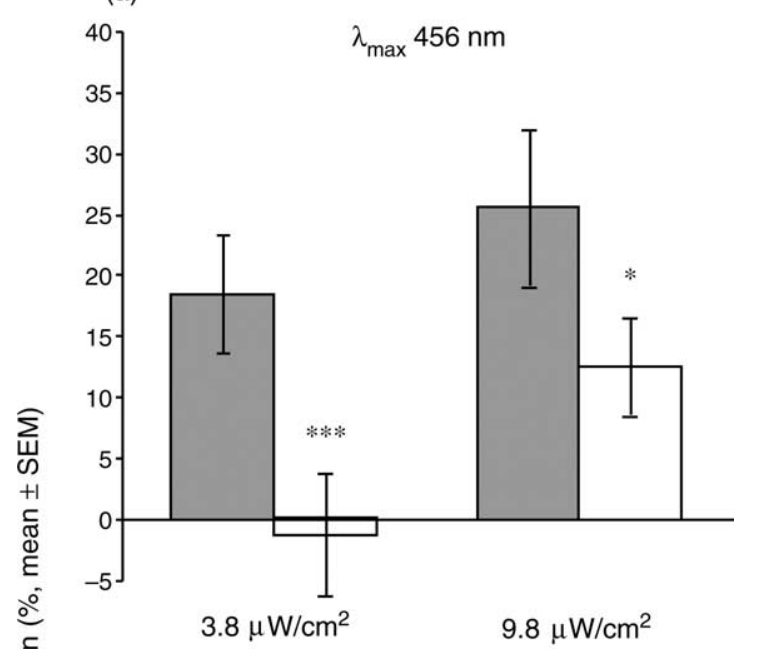

(b)

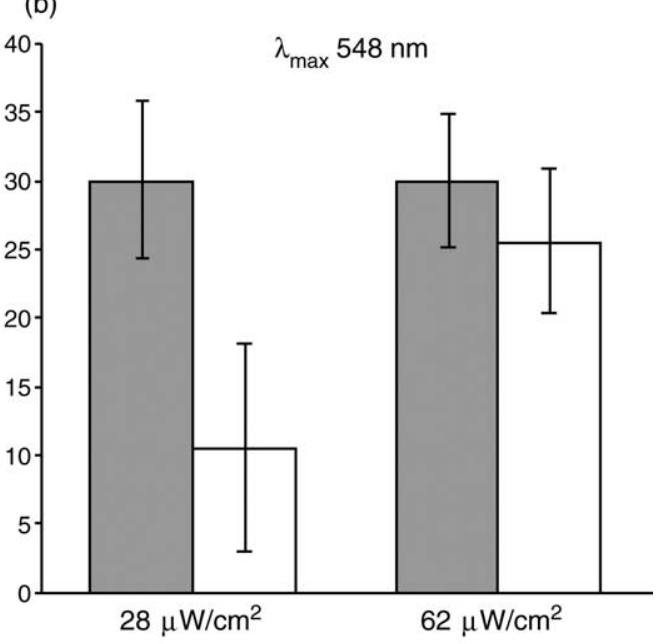

Fig. 2. Average $\%$ melatonin suppression in young $(\square, n=13)$ and postmenopausal $(\square, n=19$ ) women across the four light treatments: (a) $\lambda_{\max } 456 \mathrm{~nm}: 3.8 \mu \mathrm{W} / \mathrm{cm}^{2}$ and $9.8 \mu \mathrm{W} / \mathrm{cm}^{2}$, (b) $\lambda_{\max } 548 \mathrm{~nm}$ : $28 \mu \mathrm{W} / \mathrm{cm}^{2}$ and $62 \mu \mathrm{W} / \mathrm{cm}^{2}$. $* p<0.05 ; * * * p<0.001$ compared to the corresponding young group (Tukey HSD post hoc test).

mainly male, subjects. Whether factors such as gender and age or simply the difficulty of taking blood samples from elderly women may affect repeatability requires further study.

The present study only assessed melatonin suppression in women. Two studies (Boyce and Kennaway, 1987; Nathan et al., 2000) have shown no significant gender difference in melatonin suppression using a range of intensities (200-3000 lux) of white light. These findings were in contrast to a report which showed females had a greater sensitivity to 2000 lux of white light in comparison to males (Monteleone et al., 1995). In a preliminary study comparing the response of young men and young women to monochromatic 456 and $548 \mathrm{~nm}$ light, although the young females showed reduced melatonin suppression compared to the men, these gender differences were not statistically significant (Skene, Middleton, Thapan, unpublished data).
Thus, although it is most likely that older men would also show reduced sensitivity to short wavelength light similar to that demonstrated in older women, this remains to be shown.

The present results raise the question of the minimum amount of short wavelength light required to maintain adequate circadian synchronisation in older people. The current study provides the first experimental evidence to support the idea that the minimum levels of short wavelength light needed for photic entrainment of the circadian system would be different for young compared to older individuals. Future studies are needed to determine what these minimum levels might be and how these relate to an individual's lens density. If the ability of short wavelength light to phase shift circadian rhythms is also reduced in the elderly (and this is likely on the basis of the present results), this may have important practical implications. For example, appropriate lighting adjustments may need to be made to hospitalised and housebound elderly who may not be exposed to sufficient amount of illumination (Campbell et al., 1988; Van Someren et al., 1997; Monk et al., 2000). Furthermore, elderly shift workers may need more short wavelength light of a higher intensity than younger colleagues in order to hasten adaptation to a new shift schedule.

The light irradiances tested in the current study (3.8 and $9.8 \mu \mathrm{W} / \mathrm{cm}^{2}$ ) were 10 -fold lower than the average household illumination of $300 \mathrm{lux}\left(100 \mu \mathrm{W} / \mathrm{cm}^{2}\right)$. Even this low intensity experimental light is capable of suppressing nocturnal melatonin production, in agreement with previous studies (Brainard et al., 2001; Thapan et al., 2001). However, before short wavelength light can be used in real life in repeated doses, light-induced photochemical damage to the retina (photoretinitis), also known as blue light hazard, needs to be assessed. Blue light hazard peaks at $440 \mathrm{~nm}$ and falls to $80 \%$ at $460 \mathrm{~nm}$ (ICNIRP, 1997). Adjusting lighting to $460-480 \mathrm{~nm}$ would possibly minimise any retinal injury while still retaining the most effective short wavelengths of light necessary for circadian entrainment.

In summary, we have demonstrated reduced melatonin suppression in response to short wavelength light in older postmenopausal women. The implications of this reduced light sensitivity for photic entrainment of the circadian system in older people require further study.

\section{Acknowledgements}

We would like to thank Dr Victoria Revell and Ms Selvamalar Ratnasingam for all their help during the clinical trials. Special thanks also go to all the volunteers for their time and dedication to the project. This research was supported by an EU 5th Framework grant (QLK6-CT2000-00499). 


\section{References}

Ancoli-Israel, S., 2000. Insomnia in the elderly: a review for the primary care practitioner. Sleep 23, S36-S38.

Bliwise, D.L., 1993. Sleep in normal aging and dementia. Sleep 16, 40-81.

Boyce, P., Kennaway, D.J., 1987. Effects of light on melatonin production. Biol. Psychiatry 22, 473-478.

Brainard, G.C., Hanifin, J.P., Greeson, J.M., Byrne, B., Glickman, G., Gerner, E., Rollag, M.D., 2001. Action spectrum for melatonin regulation in humans: evidence for a novel circadian photoreceptor. J. Neurosci. 21, 6405-6412.

Campbell, S.S., Kripke, D.F., Gillin, J.C., Hrubovcak, J.C., 1988. Exposure to light in healthy elderly subjects and Alzheimer's patients. Physiol. Behav. 42, 141-144.

Coren, S., Girgus, J.S., 1972. Density of human lens pigmentation: in vivo measures over an extended age range. Vision Res. 12, 343-346.

Dijk, D.J., Duffy, J.F., 1999. Circadian regulation of human sleep and agerelated changes in its timing, consolidation and EEG characteristics. Ann. Med. 31, 130-140.

Dillon, J., Atherton, S.J., 1990. Time resolved spectroscopic studies on the intact human lens. Photochem. Photobiol. 51, 465-468.

Duffy, J.F., Dijk, D.J., Klerman, E.B., Czeisler, C.A., 1998. Later endogenous circadian temperature nadir relative to an earlier wake time in older people. Am. J. Physiol. 275, R1478-R1487.

Fraser, S., Cowen, P., Franklin, M., Franey, C., Arendt, J., 1983. Direct radioimmunoassay for melatonin in plasma. Clin. Chem. 29, 396-397.

Garner, B., Vazquez, S., Griffith, R., Lindner, R.A., Carver, J.A., Truscott, R.J., 1999. Identification of glutathionyl-3-hydroxykynurenine glucoside as a novel fluorophore associated with aging of the human lens. J. Biol. Chem. 274, 20847-20854.

International Commission on Non-Ionising Radiation Protection (ICNIRP), 1997. Guidelines on limits of exposure to broad band incoherent optical radiation. Health Phys. 73, 539-554.

Jedziniak, J.A., Kinoshita, J.H., Yates, E.M., Hocker, L.O., Benedek, G.B., 1973. On the presence and mechanism of formation of heavy molecular weight aggregates in human normal and cataractous lenses. Exp. Eye Res. 15, 185-192.

Lerman, S., Kuck Jr., J.F., Borkman, R., Saker, E., 1976. Accleration of an aging parameter (fluorogen) in the ocular lens. Ann. Ophthalmol. 8, 558-561.

Lockley, S.W., Brainard, G.C., Czeisler, C.A., 2003. High sensitivity of the human circadian melatonin rhythm to resetting by short wavelength light. J. Clin. Endocrinol. Metab. 88, 4502-4505.
Monk, T.H., Buysse, D.J., Carrier, J., Kupfer, D.J., 2000. Inducing jet-lag in older people: directional asymmetry. J. Sleep Res. 9, 101-116.

Monteleone, P., Esposito, G., La Rocca, A., Maj, M., 1995. Does bright light suppress nocturnal melatonin secretion more in women than men? J. Neural. Transm. Gen. Sect. 102, 75-80.

Nathan, P.J., Burrows, G.D., Norman, T.R., 1999. The effect of age and prelight melatonin concentration on the melatonin sensitivity to dim light. Int. Clin. Psychopharmacol. 14, 189-192.

Nathan, P.J., Wyndham, E.L., Burrows, G.D., Norman, T.R., 2000. The effect of gender on the melatonin suppression by light: a dose response relationship. J. Neural. Transm. 107, 271-279.

Norren, D.V., Vos, J.J., 1974. Spectral transmission of the human ocular media. Vision Res. 14, 1237-1244.

Pokorny, J., Smith, V.C., Lutze, M., 1987. Aging of the human lens. App. Optics 26, 1437-1440.

Said, F.S., Weale, R.A., 1959. The variation with age of the spectral transmissivity of the living human crystalline lens. Gerontologia 3, 213-231.

Skene, D.J., Swaab, D.F., 2003. Melatonin rhythmicity: effect of age and Alzheimer's disease. Exp. Gerontol. 38, 199-206.

Smith, V.C., Pokorny, J., 1975. Spectral sensitivity of the foveal cone photopigments between 400 and $500 \mathrm{~nm}$. Vision Res. 15, 161-171.

Thapan, K., Arendt, J., Skene, D.J., 2001. An action spectrum for melatonin suppression: evidence for a novel non-rod, non-cone photoreceptor system in humans. J. Physiol. 535, 261-267.

Van Someren, E.J., Kessler, A., Mirmiran, M., Swaab, D.F., 1997. Indirect bright light improves circadian rest-activity rhythm disturbances in demented patients. Biol. Psychiatry 41, 955-963.

Van Someren, E.J., 2000. Circadian and sleep disturbances in the elderly. Exp. Gerontol. 35, 1229-1237.

Verriest, G., 1971. Influence of age on visual functions in humans. Bull. Acad. R. Med. Belg. 11, 527-578.

Warman, V.L., Dijk, D.J., Warman, G.R., Arendt, J., Skene, D.J., 2003. Phase advancing human circadian rhythms with short wavelength light. Neurosci. Lett. 342, 37-40.

Weale, R.A., 1954. Retinal irradiation and aniseikonia. Br. J. Ophthalmol. 38, 248-249.

Weale, R.A., 1985. Human lenticular fluorescence and transmissivity, and their effects on vision. Exp. Eye Res. 41, 457-473.

Xu, J., Pokorny, J., Smith, V.C., 1997. Optical density of the human lens. J. Opt. Soc. Am. A. 14, 953-960.

Yu, N.T., Denagel, D.C., Pruett, P.L., Kuck Jr., J.F., 1985. Disulfide bond formation in the eye lens. Proc. Natl Acad. Sci. USA 82, 7965-7968. 